\title{
Identification of Cool White Dwarfs in the Sloan Digital Sky Survey
}

\author{
T. S. Metcalfe, A. Mukadam, D. E. Winget
}

Department of Astronomy, University of Texas, Austin, TX 78712, USA

\author{
X. Fan, M. A. Strauss \\ Department of Astrophysical Sciences, Princeton University, Princeton, \\ NJ 08544, USA \\ C. Claver \\ National Optical Astronomy Observatories, 950 North Cherry Avenue, \\ Tucson, AZ 85726, USA
}

\begin{abstract}
We are searching for the coolest white dwarf stars in the galactic disk and halo. The Sloan survey, in due course, will identify an enormous number of new white dwarf stars which will better define the white dwarf luminosity function-an important tool for understanding the age and history of the stellar population of the galaxy. The broadband filter data obtained in the digital photometry phase of the survey will not permit identification of the most interesting of these, the coolest white dwarf stars. This is because the cool main sequence and subdwarf stars become indistinguishable from the white dwarfs in the various colorcolor diagrams. We have interference filters designed to separate out these classes of objects. We have obtained photometry of test fields to complement the Sloan data and identify the population of cool white dwarf stars. These data will ultimately resolve the controversies, based for the most part on small-number statistics, of the location of the turndown in the white dwarf luminosity function for the disk. If the halo is significantly older than the disk, we will find a second peak in the white dwarf luminosity function, at lower luminosities than the disk turndown. Our data will provide the first meaningful constraints on the location of the turndown in the halo white dwarf luminosity function.
\end{abstract}

